\title{
EL COLERA EN COCENTAINA EN LA PRIMERA MITAD DEL SIGLO XIX. ASPECTOS DEMOGRAFICOS
}

\author{
Primitivo Pla Alberola \\ Univ. de Alicante
}

En este artículo se adelantan algunos resultados de la tesis que, sobre el condado de Cocentaina, realiza el autor, bajo la dirección de D. Antonio Gil Olcina.

El cólera ocupa, cronológicamente, el último lugar entre las grandes epidemias que han asolado de forma periódica a la población en los últimos siglos. Primero fue la peste, después la fiebre amarilla y, por último, la epidemia que, desde una óptica local, es objeto de estas páginas; de cuyos efectos, relativamente recientes, aún se guarda recuerdo por tradición oral.

El temor que estas epidemias produjeron, los testimonios que de su paso nos han legado y el interés que su estudio suscita, quizás hayan desorbitado la valoración que se da a su incidencia sobre la evolución demográfica de la sociedad. El temor a la epidemia, a la «pestilencia» de los tratadistas modernos, se debe quizás tanto como a los estragos humanos que produjo a la aleatoriedad de su aparición, al hecho de que la pérdida de vidas se concentrase en un corto lapso de tiempo, a la impotencia de las medidas curativas y la frecuente inoperancia de los intentos de frenar su desarrollo. El consejo de «huir presto y lejos» de la zona infecta fue con mucha frecuencia seguido, no sólo ante las sucesivas epidemias de peste sino también en los brotes coléricos del siglo XIX.

Recientes estudios han cuestionado ese papel determinante que a la grandes epidemias se viene concediendo en la evolución de la población. Pérez Moreda ha puesto de manifiesto como, en general, las crisis 
de mortalidad no constituyen un factor determinante que sirva para explicar el comportamiento demográfico del centro de Castilla; y como, dentro de éstas, tienen más importancia otras epidemias (1).

Para conocer la incidencia del cólera se dispone de evaluaciones oficiales. Los poderes públicos están interesados en conocer su desarrollo y gravedad, y para ello recaban información de las correspondientes Juntas de Sanidad. Los periódicos partes remitidos por las mismas, especificando el número de invadidos, fallecidos y curados, son regularmente publicados en el Boletín Oficial de la Provincia de Alicante (BOPA). Estos datos deberían permitirnos medir la gravedad que revistió la epidemia, pero su fiabilidad ha sido repetidamente cuestionada tanto a nivel nacional (2) como en diversos estudios locales (3).

Si en 1834 la publicación en el BOPA de los partes remitidos por las juntas locales de sanidad queda pronto interrumpida, antes de que conste referencia alguna al cólera en Cocentaina. En 1855 los informes transmitidos por la Junta de Sanidad de Cocentaina aparecen regularmente, en los partes de la Junta Provincial de Sanidad, desde el de 23 de agosto (BOPA $n^{\circ} 110$ ) al de 14 de septiembre (BOPA $n^{\circ} 120$ ); no constando ningún otro en los posteriores informes del desarrollo de la epidemia, ni se haga mención a que el cólera hubiese tocado a su fin en esta localidad.

En 1855, desde el 1 de agosto hasta el 8 de septiembre, ya que el último parte indica que el día 9 no hubo «ninguna novedad», fueron, según esta fuente, invadidos 98 individuos, 27 fallecieron y 40 sanaron; registrándose la última defunción el 6 de septiembre. La confrontación con los datos anotados en las parroquias nos da idea de la importancia del error. La diferencia es considerable; 27 óbitos según el BOPA, 112 según los registros parroquiales; aquél sólo da cuenta del $24 \%$ de las defunciones imputables al cólera. Aun teniendo en cuenta que el período efectivamente cubierto por los partes que publica el BOPA comprende desde el 1 de agosto al 9 de septiembre (faltando datos del

(1) PEREZ MOREDA, V.:Las crisis de mortalidad en la España interior (siglos XVI$X(X)$; Siglo XXI, Madrid 1980.

(2) NADAL, J.: La población española (siglos XVI a XX); Editorial Ariel, Barcelona, 1976, pp. 151 y ss.

(3) ARACIL, R., BERNABE, J.M. i GARCIA, M.: "Les epidèmies colèriques a Alcoi: 1854 i 1855); ; en Primer Congreso de Historia del País Valenciano, vol. IV, Univérsidad de Valencia, Valencia 1974, pp. 375-6. 
día 4 del mismo mes), los fallecidos a causa del cólera cifrados por el BOPA representan sólo el $\mathbf{2 8} \%$ de los registrados en las parroquias; las diferencias no se explican únicamente por las posibles lagunas sino por la calidad de la información, totalmente recusable.

El importante error de las cifras oficiales hace necesario recurrir a las fuentes locales, a los libros parroquiales, para poder estudiar adecuadametne los efectos del cólera. No es que estén sus datos a salvo de toda crítica, es conocido como en momentos de sobremortalidad, y por distintas causas, buen número de los fallecidos podían no ser anotados en los libros de las sepulturas; Navarro Martínez atribuye el escaso número de óbitos que se registran en Enguera en 1855 a que «hay que tener en cuenta la falta de datos reales en los Libros Parroquiales» (4). No hay ningún elemento - desorganización del registro, lagunas, encaballamientos de actas, abandono de las parroquias por sus titulares - que permitan suponer en Cocentaina una omisión más allá de la imputable a causas puramente fortuitas. El registro es llevado de una forma escrupulosa y se deja constancia, incluso, de los muertos en el acto de nacer.

En las parroquias de Cocentaina, en las actas de defunción, los encargados del régistro hacen constar, en buena parte del siglo XIX, unos datos preciosos para el estudio del cólera: la edad del fallecido y las causas de la defunción, además de los habituales en otros períodos. Estos datos están completos, salvo omisiones ocasionales, en ambas parroquias para los años 1854 y 1855; en 1834, en cambio, faltan en la parroquia de El Salvador, en todas las actas, las indicaciones de la causa de la defunción que permitan aislar las debidas al cólera en el total de los fallecidos, y con la misma dificultad se tropeiza en las actas de «albats» de la parroquia de Santa María en ese mismo año. (5).

Otra cuestión es la de la calidad de estos datos, edad y causa de la defunción. Es conocida la tendencia a redondear las edades declaradas. Una sumaria comprobación nos la ofrece el cuadro I, en el que se recogen as terminaciones de las edades declaradas por los fallecidos de cólera en los años 1854 y 1855 . Queda de manifiesto como se tienden a declarar edades aproximadas a la decena, de forma más palpaple cuan-

(4) NAVARRO MARTINEZ, N.: "Demografía de Enguera durante el siglo $X I X »$; en Primer Congreso de Historia del País Valenciano, vol. IV, Universidad de Valencia, Valencia, 1974, p. 181.

(5) En conjunto, estas anotaciones cubren con suficiente continuidad el periodo comprendido entre fines de 1849 a fines de 1855 y desde principios de 1858 en adelante en el registro de "albats", y en el de adultos se anotan las causas de la defunción desde 1819, todo ello en la parroquia de Santa María. En la parroquia de El Salvador, donde se registran conjuntamente "albats» y adultos, constan estos datos desde mediados de 1806 a mediados de 1809, y desde 1838 en adelante. 
to mayor es la edad del fallecido; redondeo que además parece realizarse sobreevaluando la edad. Sobre la fiabilidad del dato que más nos interesa aquí, la causa de la defunción, toda comprobación es difícil, nunca se podrá asegurar la calidad del diagnóstico; si que es interesante resaltar que no se presentan estos registros de las deficiencias que se dan en las localidades pequeñas, pareja a la insuficiente cobertura de la asistencia médica (6).

\section{CUADRO I}

\section{Edad del difunto}

\section{Terminaciones en}

$\begin{array}{lcccccc} & \frac{0}{15^{\prime} 2} & \frac{1}{6^{\prime} 4} & \frac{2}{16} & \frac{3}{16} & \frac{4}{14^{\prime} 4} & \frac{5}{7^{\prime} 2} \\ \text { 0-49 años } & 34^{\prime} 9 & 3^{\prime} 6 & 12^{\prime} 1 & 6 & 13^{\prime} 3 & 9^{\prime} 6 \\ 50 \text { y más } & 23 & 5^{\prime} 3 & 14^{\prime} 4 & 12 & 14 & 8^{\prime} 2 \\ \text { Total } & 6 & \frac{7}{3} & \frac{8}{7 \prime 2} & \frac{9}{4} & & \frac{\text { Total }}{} \\ 0-49 \text { años } & 9^{\prime} 6 & 4 & 7^{\prime} 2 & 4 & & 100 \\ 50 \text { y más } & 12^{\prime} 1 & 2^{\prime} 4 & 6 & 0 & & 100 \\ \text { Total } & 10^{\prime} 6 & 3^{\prime} 4 & 6^{\prime} 7 & 2^{\prime} 4 & & 100\end{array}$

Según las indicaciones de los registros parroquiales, el cólera hace su aparición en Cocentaina, hasta 1859 inclusive, en los años 1834,1854 y 1855; costando en julio de 1851, en la Parroquia de Santa María, un caso aislado que figura como "cólera espontáneo». En cambio, el cólera que afectó a la vecina localidad de Alcoy en 1859 no se extendió a Cocentaina. En los tres años citados la incidencia del cólera queda patente en el número de óbitos registrados, con aumentos sensibles en los respectivos totales anuales y saldos vegetativos negativos. En la parroquia de Santa María estos saldos negativos son los únicos que se registran en esta primera mitad del siglo XIX, además de los que tienen lugar los años 1809 y 1812-13. En cambio, en la Parroquia de El Salvador son más frecuentes los años que el número de las defunciones supera al de los nacidos (gráfico 1). Diferencias, que, sin duda, son directo reflejo de las

(6) En Gayanes, por ejemplo, por estas mismas fechas es muy frecuente que el párroco haga constar que se desconoce la causa del fallecimiento, por la ausencia de un facultativo que le prestase atención médica. 


\section{GRAFICO I}
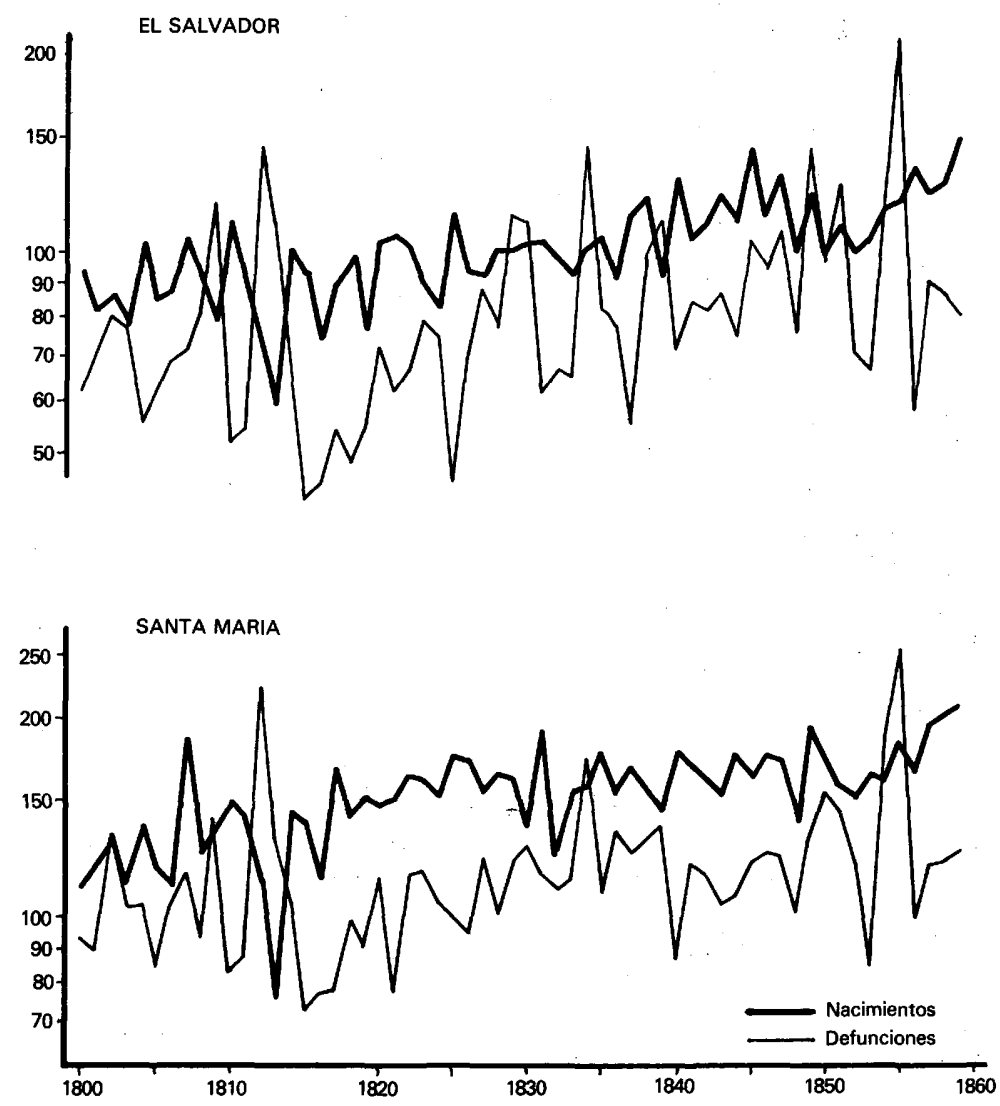

que se dan en cuanto a la categoría socioprofesional de sus respectivos parroquianos.

Interesa perfilar más la incidencia que el cólera tiene en Cocentaina a través del cálculo de las tasas de natalidad y mortalidad de esos años. La posibilidad de efectuarlo la brindan el censo de 1833 y el de 1857 , con el que se considera inaugurada la etapa estadística en el estudio de la población española.

Borja Tarrius publicó en su tiempo la «matrícula de 1833» según la 
nueva división provincial surgida del decreto de 30 de noviembre de ese mismo año (7). Hay que considerar entonces las cifras de población que ofrece como posteriores al citado decreto (para simplificar 31 de diciembre), aunque puede que recojan otras anteriores.

Este censo da 5.805 habs. para Cocentaina, a los que hay que añadir los 90 habs. de San Rafael, que está incluido en la Parroquia de Santa Maria, siendo en total 5.895 habs. Un problema que se plantea en la "matrícula de 1833" no se hace referencia a la Alcudia que, aunque en la "contribución» de Cocentaina, forma parroquia aparte con Rafol Blanch. Tomando la población de las respectivas localidades como proporcional al número de nacimientos en ellas registrados, a la Alcudia corresponderían 159 habs. y a Cocentaina, con San Rafael 5.736 habs., que el 1 de julio de 1834 serían 5.798. La fiabilidad de estas estimaciones puede considerarse como suficiente, aunque quizás algo subevaluadas, bien por error en el procedimiento o por tendencia inveterada de resistencia debida al temor fiscal; como índice de su aproximación digamos que la media de nacimientos de 1833-34 suponen una natalidad del $44^{\prime} 29 \%$.

El censo de 1857 cifra el 21 de mayo de ese año (simplificando, el 31 de mayo) la población contestana en un total de 7.369 habs. En este caso no se individualizan los datos ni de San Rafael ni de la Alcudia. Es necesario, como en 1834, restar la población que se estime para esta localidad, que, en 1857 estaría en torno a los 198 habs.; quedando la población del área de cobertura en ambas parroquias en 7.171 habs. Operando como si en los años anteriores nō hubiesen tenido lugar movimientos migratorios o, igualadas emigración e inmigración, se registrase un saldo migatorio nulo, la población no se vería modificada en sus efectivos más que por el balance de nacimientos y defunciones. Asi, el 1 de julio de 1854, la población de Cocentaina sería de 7.200 habs, y un año más tarde de 7.166 habs.

Con estos datos de población ya se pueden conocer la natalidad y mortalidad en los años de epidemia de cólera (cuadro II). (7)

CUADRO /I

\begin{tabular}{|c|c|c|c|}
\hline & 1834 & 1854 & 1855 \\
\hline Natalidad (\%o) & 45 & $38^{\prime} 6$ & $42^{\prime} 4$ \\
\hline alidad $(\% o)$ & 55 & $42^{\prime} 1$ & 64 \\
\hline Crecimiento vegetativo (\%o) & -10 & $-3^{\prime} 5$ & -22 \\
\hline
\end{tabular}

(7) Hay que hacer mención de que las tasas de mortalidad están subevaluadas en la medida que no constan las defunciones del clero regular. 
En ninguno de los tres casos se puede hablar de catástrofe demográfica. La crisis de mortalidad más aguda corresponde al año 1855, de menor entidad es la de 1834 , y en 1854 el aumento de la mortalidad está en el límite de ese aumento del $50 \%$ de sus valores ordinarios requerido para hablar propiamente de una crisis. Los déficits demográficos de estos años no suponen una quiebra notable en el desarrollo de la población, ya que son pronto subsanados por el balance positivo de los años inmediatos: -58 es el saldo bruto de $1834,+94$ el del año siguiente; los 7.097 habs. estimados para el 1 de enero de 1854, dos años después, en igual fecha de 1856, eran 6.911, los saldos negativos de estos dos años supusieron una pérdida del $2^{\prime} 6 \%$ de la población, saldos negativos enjugados antes de cumplido el tercer aniversario del último de los brotes epidémicos contemplados.

III

Una cuestión a diluciar es la responsabilidad que al cólera cabe en estas crisis de mortalidad:

- En 1834 ya se ha dicho que no se dispone de la causa de defunción más que los adultos de Santa María. En esta Parroquia los 51 difuntos por cólera se espacian entre el 13 de agosto y el 15 de octubre. Suponiendo que la distribución de los óbitos por cólera fuese en 1834 proporcional a la observada en los años 1854-55, las defunciones debidas a la epidemia serían 116 , representando el $36^{\prime} 4 \%$ del total anual y una mortalidad específica del $20 \%$.

- En 1854 son 98 los fallecidos a causa del cólera entre el 31 de agosto y el $\mathbf{2 8}$ de octubre, aunque la última fecha corresponde a un caso aislado, ya que no tenían lugar defunciones por esta causa desde el 9 del mismo mes. En total, el 32 '3\% de los fallecidos en 1854 lo son por cólera, lo que supone una mortalidad del $13{ }^{\prime} 6 \%$. Las crisis de mortalidad de estos años se pueden explicar por la sola incidencia de la epidemia de cólera.

- No ocurre lo mismo en 1855; 112 son los difuntos atribuidos al cólera, sin contabilizar un "cólico sospechoso», entre el 29 de julio y el 29 de septiembre. Si al cólera corresponden el $24^{\prime} 1 \%$ de los difuntos y esta enfermedad origina una mortalidad del $15^{\prime} 6 \%$ o - menor que la de 1834-, es evidente que la epidemia de cólera no es la única causa que explique la sobremortalidad del año. En 1855 acompaña en Cocentaina al cólera otra enfermedad epidémica que reviste una mayor gravedad: el sarampión. 148 fallecidos por sarampión representan una merma más importante que la debida al cólera, no sólo en sus cifras absolutas sino en su significación demográfica: todos son niños de hasta 9 años, es- 
tando el $77^{\prime} 5 \%$ comprendidos entre 1 y 4 años; los 40 fallecidos de 1 año de edad suponen cerca del $15 \%$ de los efectivos de partida de esa generación. El sarampión viene a comprometer, en mayor medida que el cólera, el futuro desarrollo de la población al diezmar las generaciones en sus primeros años.

\section{IV}

Por último hacer referencia a la distribución por sexo y edad de las defunciones y el desarrollo claramente estacional de la epidemia.

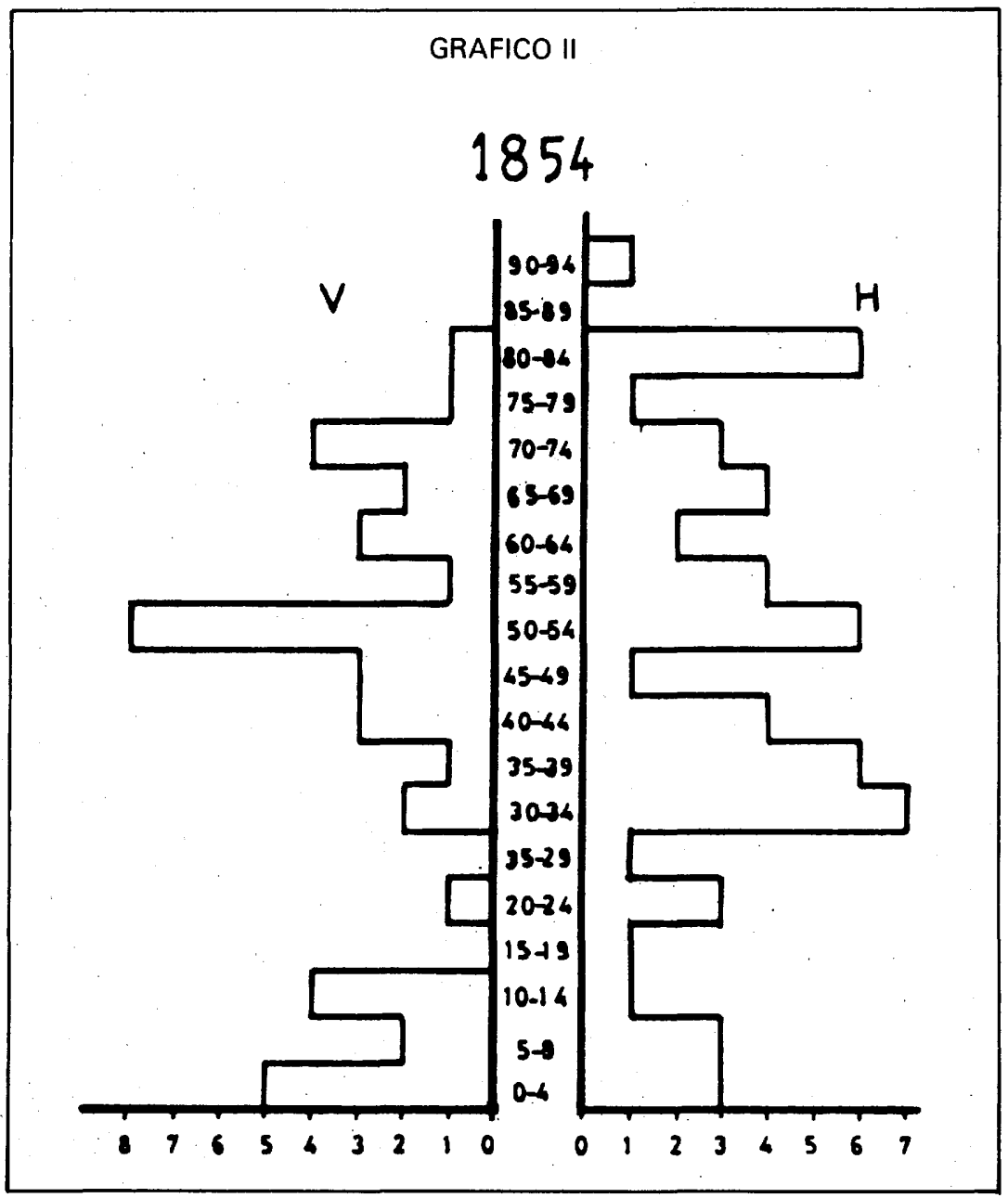




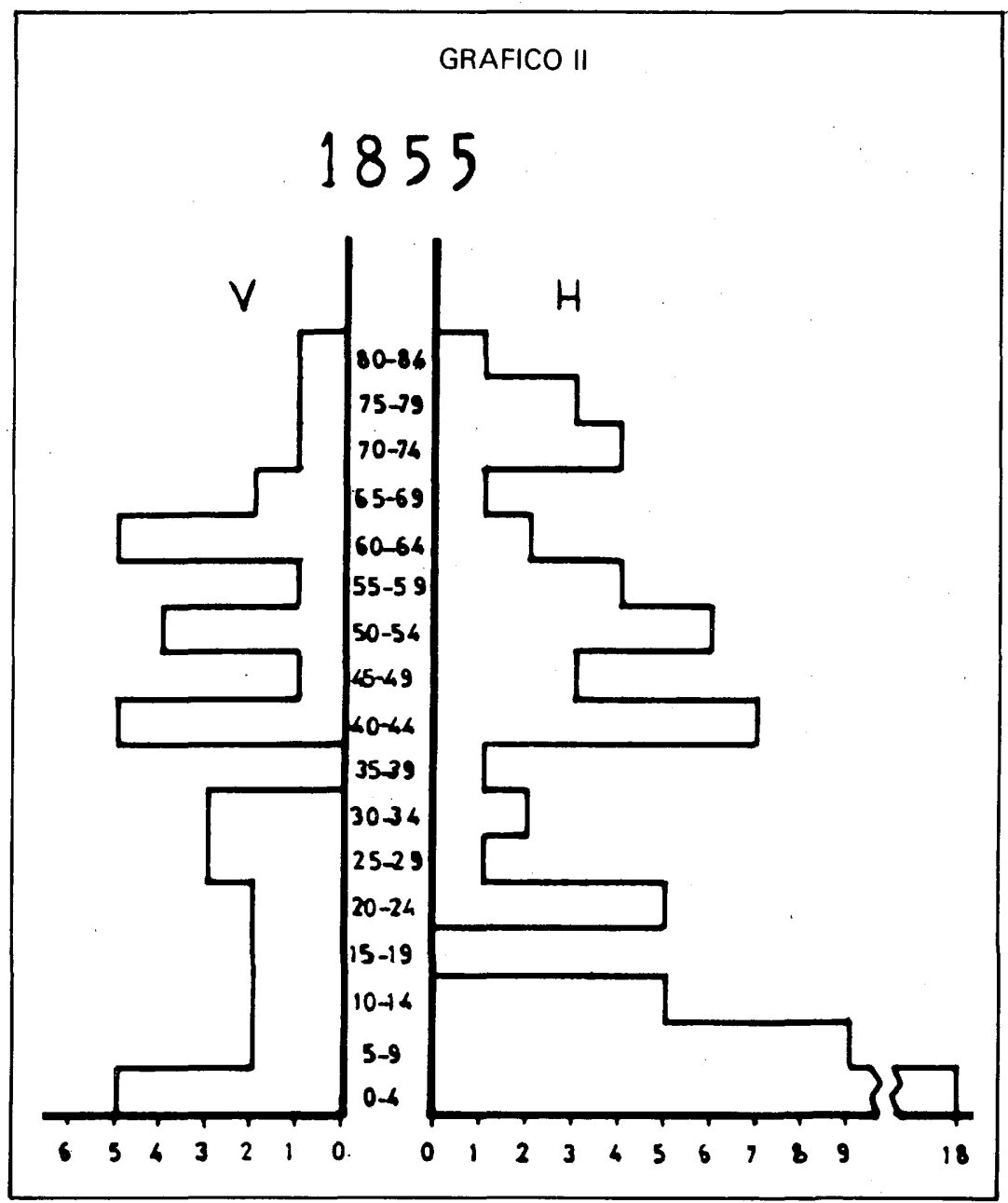

En cuanto al primero de los puntos enunciados, son muy débiles las muestras analizadas para emitir conclusiones firmes. Remitiéndose a los datos más completos de 1854-55, poner de manifiesto la acusada sobremortalidad femenina, en casi idéntica medida a la calculada por Nadal para toda España (8), por cada 100 hombres serían 159 las mujeres que murieron a causa del cólera. En lo que respecta a la edad de los falleci-

(8) NADAL, J.: Op. cit., p. 155. 
GRAFICO III

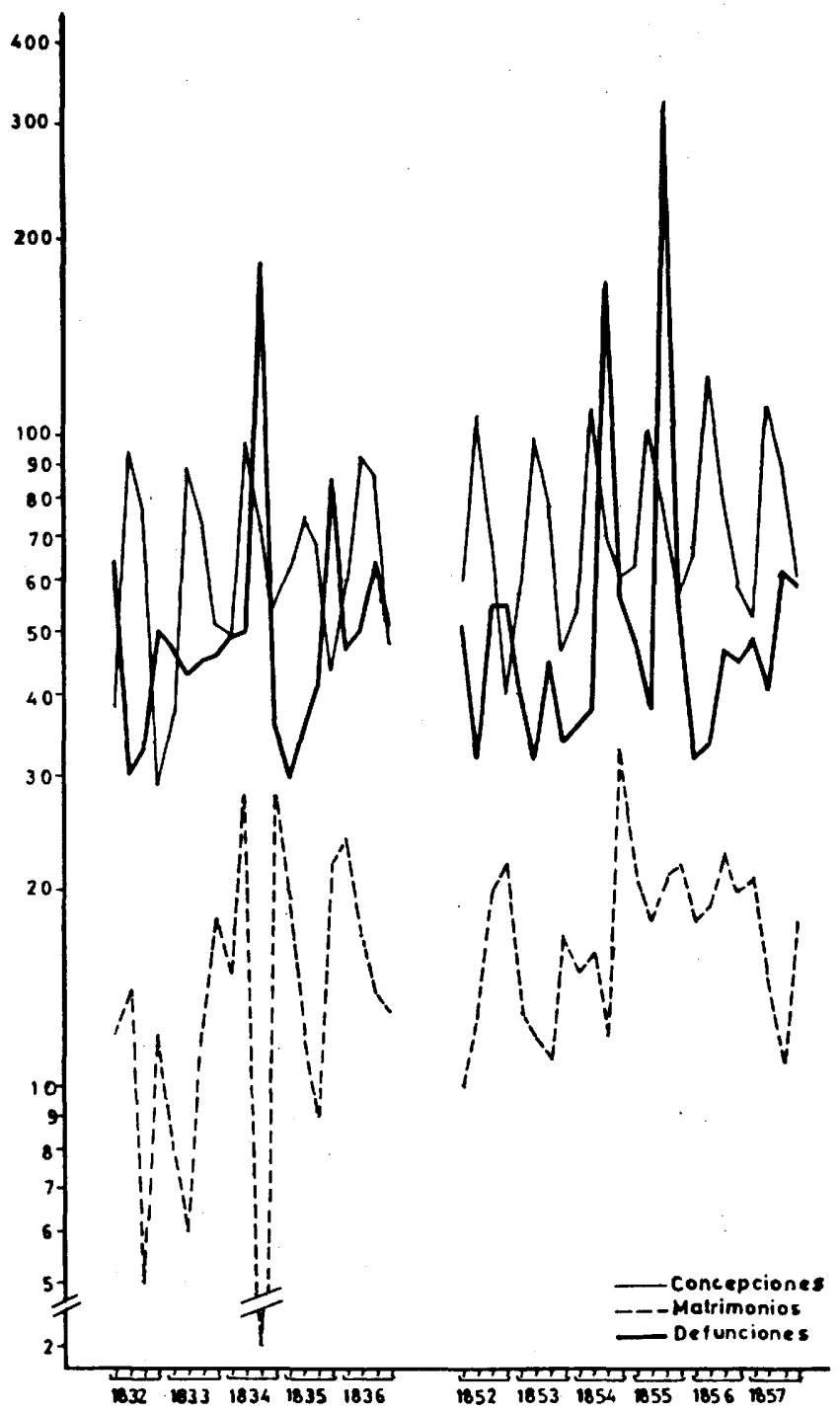


dos (gráfico II), se observa que en 1854 el cólera afecta a todos los grupos de edad, pero con una mayor incidencia entre los adultos (hay que tener en cuenta la configuración de la pirámide de edades); en 1855 las edades de los difuntos presentan una configuración semejante entre los adultos, pero existe una sobremortalidad femenina muy acusada en los dos primeros grupos de edad -27 niñas contra 7 niños - que no se da en 1854.

Los esquemas estacionales de cólera son los habituales (9), el cuadro III muestra claramente cuáles son los meses del verano y principios de otoño. Por otro lado el gráfico III, en el que se representan trimestralmente concepciones, matrimonios y defunciones, pone de manifiesto cómo el cólera no repercute en el discurrir estacional de las concepciones, que no sufren alteración apreciable (10). Otro es el caso de los matrimonios, aquí los momentos del cólera de 1834 coinciden con un descenso sustancial de celebraciones, alcanzando un mínimo señalado, mientras que en 1854 y 1855 no se registra un fenómeno similar; una vez finalizada la epidémia, en 1834 y 1854 se observa un sensible aumento del número de matrimonios en el último trimestre del año, aumento que no aparece en 1855. A partir de estos datos, y dadas las fuertes oscilaciones que registran los totales trimestrales de matrimonio, es difícil caracterizar la posible incidencia del cólera en la nupcialidad.

\section{CUADRO III}

Año

Mes

$\begin{array}{rrrrrrrrrrrrr} & E n & F b & M r & A b & M y & J n & J l & \text { Ag } & S p & O c & N v & D c \\ 1834 \ldots \ldots \ldots \ldots \ldots & 19 & 13 & 17 & 15 & 15 & 20 & 36 & 99 & 49 & 11 & 14 & 11 \\ 1854 \ldots \ldots \ldots \ldots \ldots . & 17 & 10 & 9 & 9 & 15 & 14 & 19 & 30 & 123 & 28 & 17 & 12 \\ 1855 \ldots \ldots \ldots \ldots \ldots . & 18 & 12 & 18 & 14 & 12 & 12 & 51 & 173 & 100 & 27 & 16 & 12\end{array}$

(9) PEREZ MOREDA, V.: Op. cit., pp. 396 y ss.

(10) Lo que hace suponer que el cólera no vino acompañado de una crisis de subsistencias grave que se hubiese reflejado en una disminución de las concepciones. 